\title{
A deformation based blank design method for formed parts
}

\author{
W. Hammami $\cdot$ R. Padmanabhan $\cdot$ M. C. Oliveira $\cdot$ \\ H. BelHadjSalah $\cdot$ J. L. Alves $\cdot$ L. F. Menezes
}

Received: 18 November 2008/Accepted: 11 September 2009/Published online: 23 October 2009

(C) Springer Science+Business Media, B.V. 2009

\begin{abstract}
Blank design is an important task in sheet metal forming process optimization. The initial blank shape has direct effect on the part quality. This paper presents a deformation based blank design approach to determine the initial blank shape for a formed part. The blank design approach is integrated separately into ABAQUS, and DD3IMP, a research purpose in-house FEA code, to demonstrate its compatibility with any FEA code. The algorithm uses FE results to optimize the blank shape for a part. Deep drawing simulation of a rectangular cup geometry was carried out with an initial blank shape determined empirically. The blank
\end{abstract}

W. Hammami · H. BelHadjSalah

Laboratoire de Génie Mécanique, Ecole d’Ingénieurs de Monastir, Monastir, Tunisie

e-mail: Walid.Hammami@ulg.ac.be

H. BelHadjSalah

e-mail: hedi.belhadjsalah@enim.rnu.tn

R. Padmanabhan $(\bowtie) \cdot$ M. C. Oliveira $\cdot$ L. F. Menezes

CEMUC, Centre of Mechanical Engineering

of the University of Coimbra, Polo II, Coimbra, Portugal

e-mail: padmanabhan@dem.uc.pt

M. C. Oliveira

e-mail: marta.oliveira@dem.uc.pt

L. F. Menezes

e-mail: luis.menezes@dem.uc.pt

\section{J. L. Alves}

Department of Mechanical Engineering, University of Minho, Campus de Azurém, Guimarães, Portugal e-mail: jlalves@dem.uminho.pt shape was iteratively modified, based on the deformation history, until an optimal blank shape for the part is achieved. The optimal blank shapes predicted by the algorithm using both FEA softwares were similar. Marginal differences in the shape error indicate that the deformation history based push/pull technique can effectively determine an optimal blank shape for a part with any FEA software. For the shape error selected, both procedures estimate the optimal blank shape for the part within five iterations.

Keywords Blank shape optimization -

Deep drawing - FE simulation - Push/pull technique
Abbreviations
DD3IMP
Contraction of deep-drawing 3D IMPlicit code
DD3SHAPE Contraction of deep-drawing 3D blank SHAPE optimization code
DD3TRIM Contraction of deep-drawing 3D TRIMming code
IGES Initial graphics exchange specification
NURBS Non uniform rational basis spline

\section{Introduction}

Initial blank shape is one of the important process parameters in sheet metal forming that determines the 
quality and final cost of the formed part. An optimal blank contributes to minimize forming defects such as wrinkling and tearing resulting in a good quality part. Many blank design approaches have been proposed to determine the optimum initial blank shape. A slip line field theory based method to determine optimum blank shape was described by Kuwabara and Si (1997). The method is capable of predicting an optimal blank shape within few seconds but assumes the blank material as isotropic, rigidperfectly plastic and does not deform, i.e., the thickness of the blank does not change during the deep drawing operation. A finite element method based inverse approach to determine optimum blank contour for industrial parts was described by Guo et al. (2000). The approach uses the knowledge of discretized 3D shape of the final part. The efficiency and convergence rate is dependant on the assumed initial blank. A roll-back method to predict optimum blank shapes for industrial parts was proposed by Kim et al. (2000). The deformed blank shape is compared with the target shape and necessary modification is carried out in the initial blank. The optimal blank shape for a part was obtained by Park et al. (1999) using a deformation path iteration method. In all these approaches, either the finite element mesh size was altered during the optimization procedure or only the part's flange area was considered. In this study, a blank design method based on the deformation behavior of the blank is presented. The blank design method using a push/pull technique, described in more detail in (Padmanabhan et al. 2009), is integrated with two finite element analysis codes, namely ABAQUS (Version 6.4) and DD3IMP (Menezes and Teodosiu 2000), in order to evaluate the efficiency of the method applied with different simulation tools. The procedure includes deep drawing simulations integrated with the push/ pull optimization technique, which is integrated with both codes using B-spline curve or NURBS surface interpolation, for ABAQUS and DD3IMP, respectively. The combined numerical tools are tested to prove that it can be an economical solution in reducing time and material. In order to achieve an integrated optimization procedure, the programming language PYTHON is used to apply the push/pull technique and to input the changes in geometry in ABAQUS. In DD3IMP the changes in geometry are introduced using DD3TRIM, an in-house code used to trim 3D solid finite meshes (Baptista et al. 2006). Following sections describe the push/pull optimization technique, integration of this technique with ABAQUS and DD3IMP and, finally, its application to a rectangular cup example.

\section{Blank shape optimization procedure}

The blank shape optimization procedure involves an important task of modifying the blank contour based on the deformation behaviour and the required target shape. A push/pull technique is used in this study to modify the blank shape as described below.

\subsection{Push/pull technique}

Consider a blank, with an initial contour indicated by dashed line in Fig. 1, subjected to deep drawing. Depending on the part geometry and the non-linear flow of the blank during the forming process, the contour at the end of process may take a shape different from the required contour. In Fig. 1, the final contour is shown by the continuous line, against the required contour indicated by the thick line. The set of points $\left(\mathbf{P}_{1}, \mathbf{P}_{2}, \mathbf{P}_{3}, \ldots, \mathbf{P}_{n}\right)$, defining the original surface with positions $\mathbf{X}^{\text {init }}$, may lie either inside or outside or on the target contour after deep drawing; with their positions defined by $\mathbf{X}^{\text {final }}$. The intersection of their trajectory and the target contour defines $\mathbf{X}^{\text {inter }}$, as shown in the figure.

As illustrated in the figure, the final position of point $\mathbf{P}_{1}$ is located inside the target contour, hence, the point in the new surface has to move outside the initial surface. On contrary, since the final position of point $\mathbf{P}_{2}$ is located outside the target contour, the point in the new surface has to move inside the initial surface. Point $\mathbf{P}_{3}$ does not need correction as it lies on the target contour. The knowledge of the initial position $\mathbf{X}^{\text {init }}$, the final position $\mathbf{X}^{\text {final }}$, and the intersection position $\mathbf{X}^{\text {inter }}$ enables the push/pull technique to calculate the positions of the new set of points using,

$\mathbf{P}_{i}^{*}=\mathbf{X}_{i}^{\mathrm{init}}+\left(\mathbf{X}_{i}^{\mathrm{inter}}-\mathbf{X}_{i}^{\mathrm{final}}\right), \quad$ with $i=1, \ldots, n$,

Assuming a linear trajectory for material flow during deep drawing, the vector $\mathbf{X}_{i}^{\text {inter }}-\mathbf{X}_{i}^{\text {final }}$ defines the direction and the distance to move each of the initial 
Fig. 1 Push/pull technique used to adjust flange contour
Initial

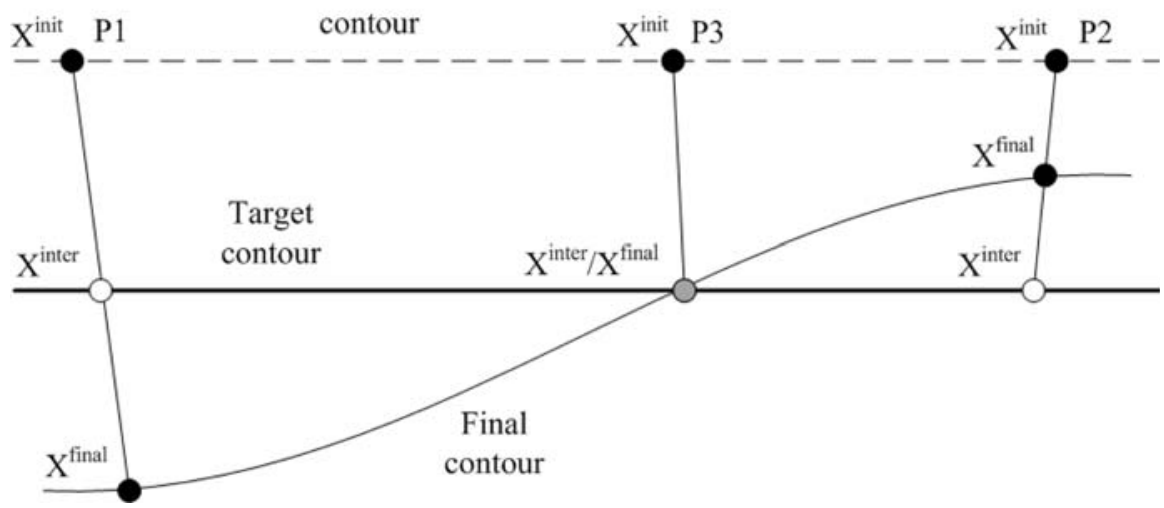

points selected. $n$ is the number of points considered along the blank contour for the push/pull technique. This push/pull technique, if applied with a damping coefficient $\xi$, controls the oscillations around the target contour that may occur due to the iterative procedure adopted. Therefore Eq. 1 can be written as

$\mathbf{P}_{i}^{*}=\mathbf{X}_{i}^{\text {init }}+\xi\left(\mathbf{X}_{i}^{\text {inter }}-\mathbf{X}_{i}^{\text {final }}\right), \quad$ with $i=1, \ldots, n$.

The objective of the optimization algorithm is to identify the difference between the existing flange contour and the required target contour and provide a corrective solution that minimizes the difference. In order to guarantee the convergence of the iterative procedure it is important that the initial process parameters like, the tools geometry, the mechanical properties of the blank, the friction conditions and the blank holder force are fixed in the numerical simulation model, during the optimization procedure. An initial blank shape is normally determined based on empirical formulae.

The optimization algorithm used in this study uses a geometric measure called geometrical shape error (GSE), to quantify the deviation between the flange and the target contours. This error, expressed in the same dimensions used for the point coordinates, is defined as the root mean square of the shape difference between the target shape and the deformed shape as in the following equation, proposed by Park et al. (1999):

$\mathrm{GSE}=\sqrt{\sum_{i=1}^{n} \frac{1}{n}\left|\mathbf{X}_{i}^{\mathrm{inter}}-\mathbf{X}_{i}^{\mathrm{final}}\right|^{2}}$.

The vector norm used corresponds to the Euclidean. The geometrical shape error defines the stopping criterion for the blank shape optimization procedure.
When the GSE reaches a value less than a predetermined value $\delta$ for the required accuracy in the flange shape, the iterative procedure is stopped because the optimal blank shape for the part has been obtained. For the example presented in this work, the error in the flange shape $(\delta)$ less than $0.5 \mathrm{~mm}$ is used as the stopping criterion.

The GSE allows correct estimation of the distance between the actual flange contour and the target contour. However, by definition it is not possible with the GSE to evaluate whether the actual flange contour is inside or outside the target contour. To clearly understand the shape error, a measure called target shape error (TSE) is used to quantify the magnitude of deviation of the flange contour from the required target contour. The TSE, expressed in the same dimensions used for point coordinates, is defined as:

$\mathrm{TSE}=\frac{1}{n} \sum_{i=1}^{n}\left[\operatorname{sign}\left(1-\frac{\left|\mathbf{X}_{i}^{\text {init }}-\mathbf{X}_{i}^{\text {final }}\right|}{\left|\mathbf{X}_{i}^{\text {init }}-\mathbf{X}_{i}^{\text {inter }}\right|}\right)\left|\mathbf{X}_{i}^{\text {init }}-\mathbf{X}_{i}^{\text {final }}\right|\right]$

where the sign function is used to identify whether a point is inside or outside the target contour. The target shape error is used mainly to analyze the convergence rate towards the solution, since it allows detecting oscillations around the target contour.

\subsection{Implementation of push/pull technique} with FEM simulations

\subsubsection{ABAQUS}

The contour of the initial blank is defined using a three degree B-spline curve, which is divided by a set of $n$ control points, as presented in Fig. 2. The $n$ 


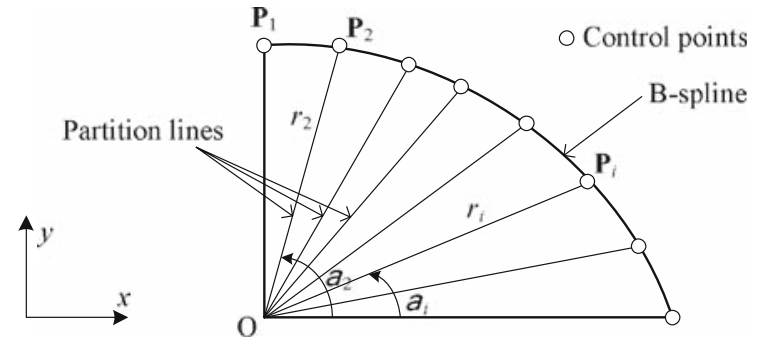

Fig. 2 Model used to implement the push/pull technique in ABAQUS

control points are selected for the initial blank shape, normally deduced empirically. These points are used to divide the blank surface along $n$ - 1 radial segments, defined by the $r_{i}, i=1, \ldots, n-1$ radial directions. The use of these control points allows changing the shape of the blank contour, in each iteration, and automatically producing the mesh for the new blank. The coordinates of the $n$ control points are changed, in each iteration, applying the push/pull technique explained previously and implemented in ABAQUS through PYTHON. This procedure alters each partition line $r_{i}, i=1, n-1$ rendering the new blank geometry. The new coordinates of each control point, determined using the push/pull technique are input to ABAQUS. ABAQUS updates the three degree Bspline curve automatically, based on the control points defined. The outer boundary of the blank geometry is described by the B-spline curve (see Fig. 2). The definition of the B-spline curve allows automatic meshing of the blank surfaces. The resulting mesh is subjected to deep drawing simulation in ABAQUS FEA code. The flange contour of the formed part is compared with the required target contour. If the flange contour is different from target contour, the initial B-spline is corrected and a new set of $n-1$ surfaces are produced. These new surfaces are used to produce intermediate blank shape with a new mesh, which is subjected to deep drawing process. The application of the push/pull technique to the surfaces based on B-spline allows guaranteeing a smooth blank contour, in each iteration.

The programming language PYTHON is used to input the data into ABAQUS, leading to an automatic blank shape optimization procedure implemented with the commercial software. The blank shape optimization procedure is illustrated in Fig. 3.

As illustrated in Fig. 3, this procedure is repeated until the deviation between flange contour and target

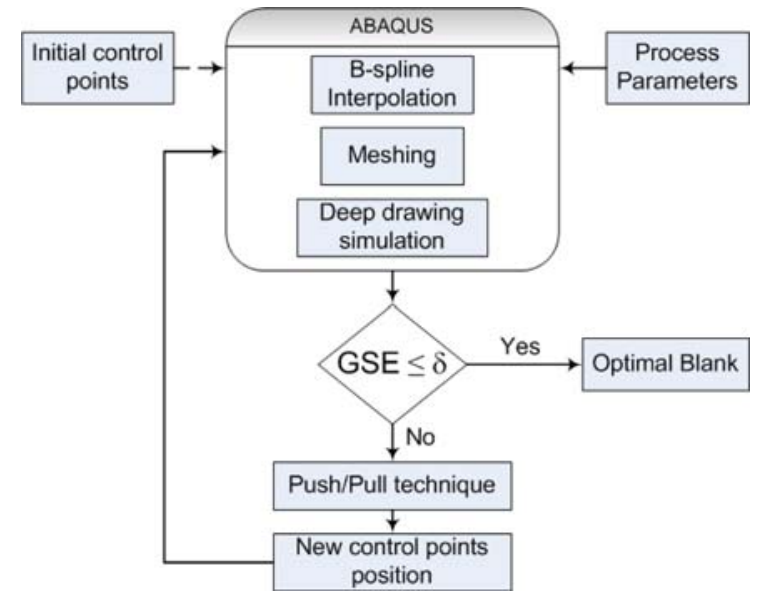

Fig. 3 Blank shape optimization procedure in ABAQUS

contour falls below the user defined value, $\delta$. The optimization procedure is fully automated due to the combination of ABAQUS and PYTHON.

\subsubsection{DD3IMP}

Three numerical tools, DD3IMP, DD3TRIM and DD3SHAPE are used in this procedure to determine the optimal blank shape for a part. Deep drawing simulations were carried out using the in-house finite element code DD3IMP. DD3IMP is specifically developed to simulate sheet metal forming processes. The evolution of the deformation process is described by an updated Lagrangian scheme. A fully implicit algorithm is used to guarantee the satisfactory equilibrium of the deformable body, in each increment, as described by Menezes and Teodosiu (2000). In sheet metal forming processes, the boundary conditions are dictated by the contact established between the blank sheet and tools. Such boundary conditions are continuously changing during the forming process, increasing the importance of a correct evaluation of the actual contact surface and the kind of contact that is established in each point of the deformable body. A master-slave algorithm is adopted, with the tools behaving as rigid bodies. The Coulomb's classical law models the friction contact problem between the rigid bodies (tools) and the deformable body (blank sheet). The problem of the contact with friction is treated by an augmented Lagrangian approach. The abovementioned fully implicit Newton-Raphson scheme is used to solve, 
in a single loop, all the problem non-linearities associated to the problem of contact with friction and the elasto-plastic behavior of the deformable body. Further details about the numerical strategies adopted in DD3IMP can be obtained from the work of Oliveira et al. (2008).

The blank shape optimization procedure using DD3IMP is illustrated in Fig. 4. An initial blank shape is defined and a corresponding NURBS surface is produced, which defines the blank contour. A regular and uniform mesh with dimension large enough to accommodate the probable blank shapes is produced and defined as base mesh.

Generally, to accommodate the continuous variation of the nodal coordinates in optimization procedure, a time-consuming remeshing procedure is employed. In the integration of the push/pull technique with DD3IMP, the base mesh is always used to produce the initial and intermediate blank shapes in accordance with the initial NURBS surface and those created by the algorithm, respectively. In each case the base mesh is trimmed by the current NURBS surface, using DD3TRIM program. This minimizes the influence of blank discretization i.e., the FE mesh variability on the optimization procedure. The mesh cut using the NURBS surface is subjected to deep drawing simulation. The flange contour of the formed part is compared with the required target contour. If the flange contour is different from target contour, the initial NURBS surface is corrected and a new NURBS surface is produced depending on its deviation as described below.

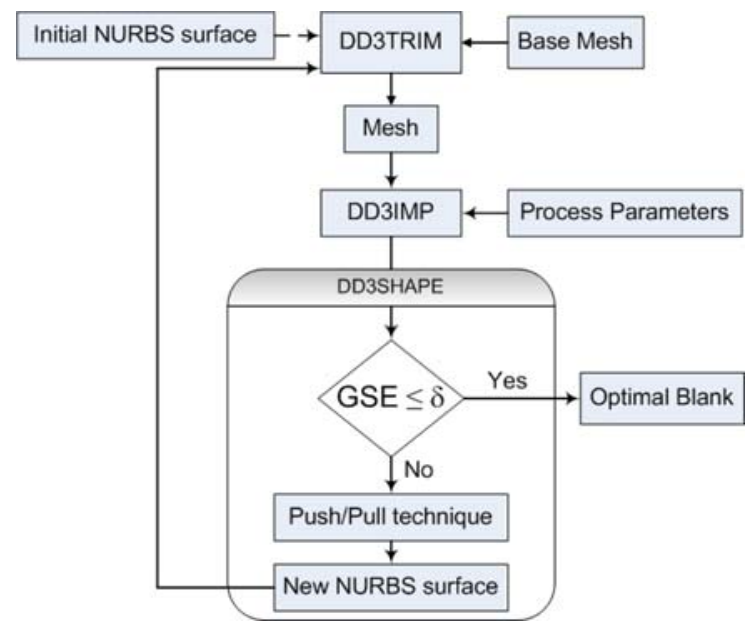

Fig. 4 Blank shape optimization procedure in DD3IMP
NURBS are the most commonly used parametric surfaces for geometric modelling. Generalized NURBS surface is preferred over other surfaces in geometric modelling because its evaluation is computationally stable and its shape can be easily changed through the manipulation of control points, weights or knots (Piegl and Tiller 1997).

Suppose an initial NURBS curve constructed using an initial set of control points $\left\{\mathbf{P}_{k}^{i}\right\}, k=0, \ldots, n-1$. Based on the deformation behaviour of the blank during deep drawing and consequently the blank shape optimization procedure, new positions for these control points are determined using the push/pull technique, illustrated in Fig. 5. In this case, the first step required to apply the push/pull technique is to identify the closest nodes of the trimmed mesh to each of the control points $\left\{\mathbf{P}_{k}^{i}\right\}, k=0, \ldots, n-1$, that defines the initial NURBS surface. The initial $\mathbf{X}^{\text {init }}$ and the final $\mathbf{X}^{\text {final }}$ coordinates of these nodes are used to determine intersection points $\mathbf{X}^{\text {inter }}$ on the target contour. The set of points $\left\{\mathbf{Q}_{k}\right\}, k=0, \ldots, n-1$ that the new NURBS surface must interpolate are determined using the push/pull technique defined by

$$
\begin{aligned}
& \mathbf{Q}_{k}=\mathbf{X}_{k}^{\text {init }}+\xi\left(\mathbf{X}_{k}^{\text {inter }}-\mathbf{X}_{k}^{\text {final }}\right) \\
& \quad \text { with } k=0, \ldots, n-1 \text { and } \xi \in[0,1]
\end{aligned}
$$

where $\xi$ is the damping coefficient used to control the push/pull optimization procedure. The new set of points is interpolated with a $p$ th-degree nonrational B-spline curve. Assigning a parameter value $\bar{u}_{k}$ to each $\mathbf{Q}_{k}$ will result in a system of $n$ linear equations,

$$
\begin{aligned}
\mathbf{Q}_{k} & =C\left(\bar{u}_{k}\right)=\sum_{i=0}^{n-1} N_{i, p}\left(\bar{u}_{k}\right) \mathbf{P}_{i}, \\
k & =1, \ldots, n-2
\end{aligned}
$$

for each direction of the Cartesian coordinate system. The chord length method is used for choosing $\bar{u}_{k}$. Let $d$ be the total chord length,

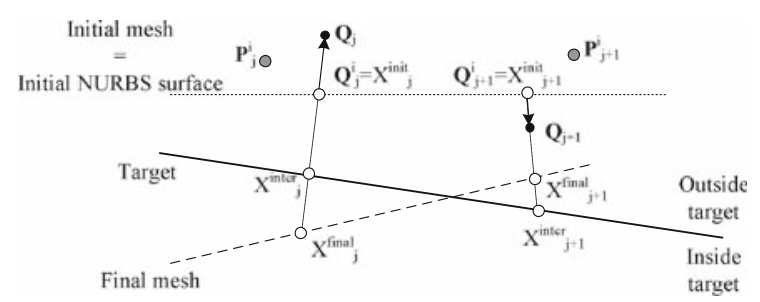

Fig. 5 Application of the push/pull technique in DD3SHAPE 
$d=\sum_{k=1}^{n-1}\left|\mathbf{Q}_{k}-\mathbf{Q}_{k-1}\right|$.

Then $\bar{u}_{0}=0, \bar{u}_{n-1}=1$, and the others are calculated using

$\bar{u}_{k}=\bar{u}_{k-1}+\frac{\left|\mathbf{Q}_{k}-\mathbf{Q}_{k-1}\right|}{d} \quad k=1, \ldots, n-2$.

Solving the system of linear equations will result in a new set of control points defining a new NURBS curve. This curve conforms to a new blank shape and it is extruded to a NURBS surface to demarcate trimming domains for the solid finite element mesh. This procedure is carried out by an in-house code named DD3SHAPE that uses the results from DD3IMP and the current NURBS surface in IGES format as inputs to produce the new surface, also in IGES format. This new NURBS surface is used to trim the base mesh to produce intermediate blank shape which is subjected to the deep drawing process. The application of push/pull technique to the NURBS surface guarantees a smooth blank contour, in each iteration. This procedure is repeated until the deviation between flange contour and target contour falls below the user defined value, $\delta$. The optimization procedure can be fully automated due to the combination of DD3IMP, DD3TRIM and DD3SHAPE (the push/pull technique applied to the NURBS surface).

\section{Rectangular cup example}

The forming tools geometry for rectangular cup used in this study is presented in Fig. 6. The desired flange shape for the rectangular cup is also presented in the figure. Numerical simulations were carried out for a quarter of the geometry due to symmetry of the part. Thus, the target contour can be defined by three lines: straight line equations (i) $\mathrm{y}=30$; for $\mathrm{x}<26$ and (ii) $\mathrm{x}=45$; for $\mathrm{y}<11$ and the circle equation (iii) $[(\mathrm{x}-$ $\left.26)^{2}+(y-11)^{2}=(19)^{2}\right]$. Only the first quadrant of the circle equation is considered for computing deviations across line 2. These lines are used in the algorithm to define the target contour. In industrial applications, the flange contour may not constitute simple geometries, in which case the DD3SHAPE algorithm takes a NURBS curve defining the required target contour as the reference.

The initial blank shape is determined based on the empirical formulae (Barata da Rocha and Ferreira Duarte 1993), and a corresponding B-spline/NURBS is produced. The B-spline curve adopted to perform the blank shape optimization with ABAQUS considers eight control points, leading to a mesh partition in seven triangular regions. The initial NURBS curve used in this study with DD3IMP program uses 23 control points evenly distributed on the contour, because previous studies indicate that the number of control points and their location density in the contour has significant influence on the error prediction (Oliveira et al. 2009).

With DD3IMP, the blank is discretized with 3D 8node solid finite elements which allow the accurate evaluation of the contact forces through an accurate description of contact evolution and thickness change; the simultaneous contact on both sides of the sheet is naturally solved without any particular strategy or algorithms. A base mesh is produced with
Fig. 6 Forming tools geometry and the desired shape

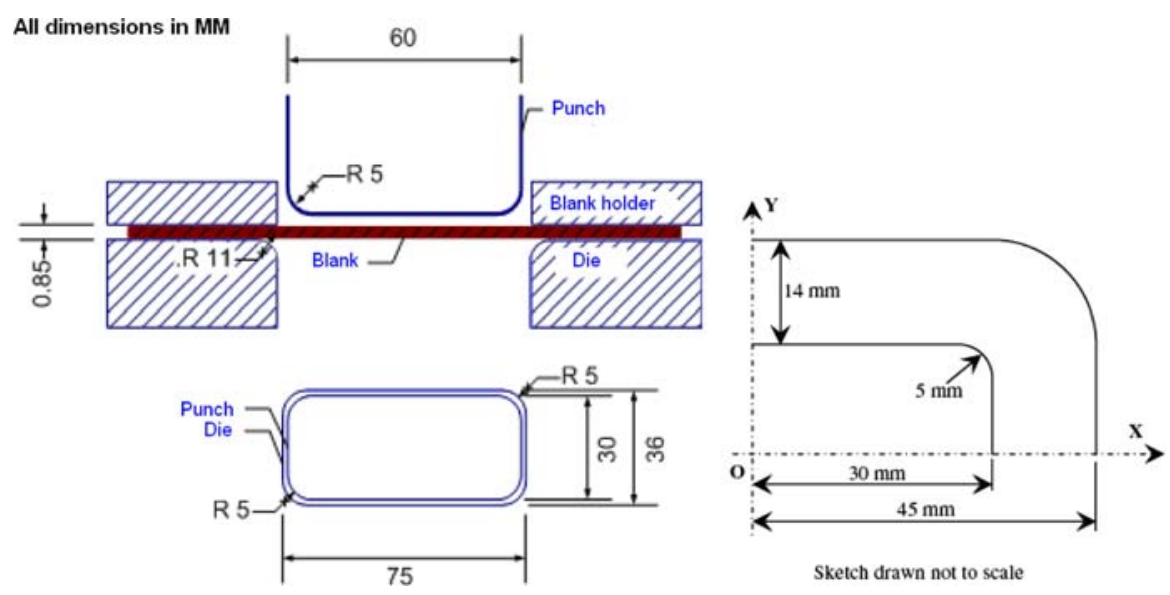


an average in-plane element size of $1.4 \mathrm{~mm}$ of eight node solid finite elements associated with selective reduced integration procedure ( $\bar{B}$ method). The blank is meshed with two layers of elements through thickness, leading to four through-thickness integration points. The average in-plane element size (turning angle $<10^{\circ}$ of die radius) guarantee accurate results with the solid element for the forming phase (Li et al. 2002). Since the selected geometry presents small springback values, the two layers of elements lead to accurate results (Oliveira et al. 2008). This base mesh is cut with initial NURBS surface to produce the initial finite element mesh. In ABAQUS, the finite elements used are 4 node fully integrated linear tetrahedron finite elements (C3D4). Tetrahedron finite elements are required due to the shape of the regions to remesh during the optimization procedure (shown in Fig. 2). However, to accurately perform structural analysis with this type of finite elements a large number is required. Figure 7 presents the initial blank shape obtained using empirical formulae and the corresponding initial inplane mesh for ABAQUS and DD3IMP.

Stainless steel (AISI304) blank of $0.85 \mathrm{~mm}$ thickness is used in this study. The material follows isotropic Hooke's law in the elastic domain, for which it is necessary to define the Young's modulus, $E$, and the Poisson's ratio, $v$. The blank shape optimization procedure described in Sect. 2.2 captures the material flow characteristics into account since it is based on the FE simulation results. The material flow characteristic is in turn governed by the initial anisotropy in addition to other material properties, in particular the work hardening. A good description of the anisotropy in the rolled sheet is essential for a quantitative approach to deep-drawing process simulations. Phenomenological plasticity theories consider initial anisotropy through an yield criterion. A number of such yield criterions have so far been proposed, while Hill (1948) is mostly preferred, due to its simplicity. Hill'48 yield function is widely used in finite element simulation studies and has been the primary choice for explaining some phenomena associated with anisotropic plasticity, especially for steel blanks. Hence in this work, the anisotropy of the rolled blank is described by the analytical function in the quadratic form postulated by Hill is given below:

$$
\begin{aligned}
\bar{\sigma}^{2}= & F\left(\sigma_{22}-\sigma_{33}\right)^{2}+G\left(\sigma_{33}-\sigma_{11}\right)^{2} \\
& +H\left(\sigma_{11}-\sigma_{22}\right)^{2}+2 L \sigma_{23}^{2}+2 M \sigma_{31}^{2}+2 N \sigma_{12}^{2}
\end{aligned}
$$

where the numerals $1,2,3$ stand for the orthotropic directions. $F, G, H, L, M, N$ are the six material parameters that depend on the flow stresses under uniaxial and shear loading in the principal directions, respectively, and implicitly assumes that hydrostatic stress states lead to no yielding and no plastic deformation. The isotropic work hardening is described using the Swift law:

$Y=K\left(\varepsilon_{0}+\bar{\varepsilon}^{p}\right)^{n}$

where $\bar{\varepsilon}^{p}$ is the equivalent plastic strain and $Y$ is the flow stress. $Y_{O}$ is the yield stress and $n$ and $K$ are the material parameters of the Swift law. All the material parameters used are listed in Table 1.

Initial process parameters were chosen based on empirical relations and optimal values. Blank holder force of $9287 \mathrm{~N}$ and friction coefficient of 0.08 was used for a draw depth of $14 \mathrm{~mm}$. These process parameters remained the same through the optimization procedure.
Fig. 7 Initial blank shape obtained using empirical formulae and the corresponding initial inplane mesh for a ABAQUS and b DD3IMP

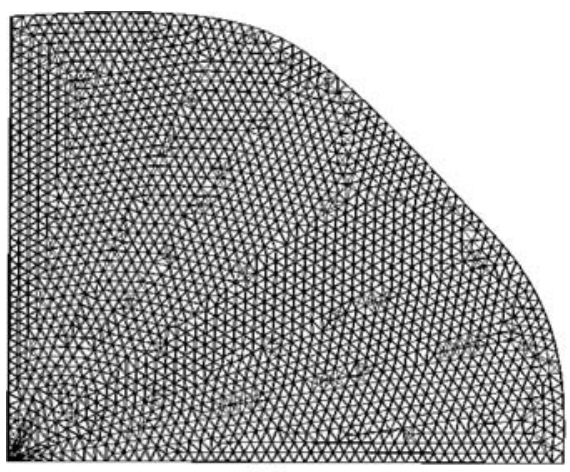

(a)

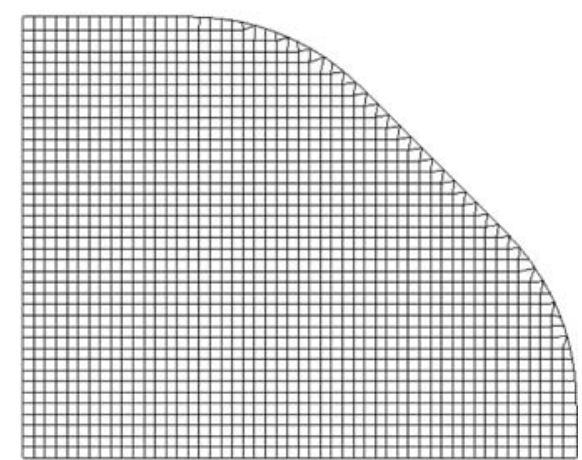

(b) 
Table 1 Constitutive parameters of stainless steel (AISI304)

\begin{tabular}{lcll}
\hline Elastic properties & \multicolumn{2}{c}{$\begin{array}{c}\text { Orthotropic behavior } \\
\text { (Hill 1948) }\end{array}$} \\
$E$ & $210.0 \mathrm{GPa}$ & $F$ & 0.46131 \\
$v$ & 0.3 & $G$ & 0.44643 \\
Isotropic hardening (Swift law) & $H$ & 0.55360 \\
$Y_{0}$ & $123.6 \mathrm{MPa}$ & $N$ & 1.35253 \\
$K$ & $529.5 \mathrm{MPa}$ & $L$ & 1.5 \\
$n$ & 0.268 & $M$ & 1.5 \\
\hline
\end{tabular}

\section{Discussion on results}

Large initial blank shape results in large TSE in the first iteration as shown in Fig. 8, and hence the correction algorithm underestimates the first intermediate blank shape for a damping coefficient of 1.0. In the second iteration, due to much smaller flange contour compared to the required target contour, the correction algorithm overestimates the second intermediate blank shape. This variation in the intermediate blank shapes increases the number of iterations to achieve the required accuracy, especially for large draw-in and deeper parts (Oliveira et al. 2009). Hence, the damping coefficient presented in Eq. 2 was used to control the variation in intermediate blank shapes, the results of which is shown in Fig. 8. The results on the influence of the damping coefficient presented in Fig. 8 were obtained with the optimization procedure implemented in DD3IMP.

The oscillation between positive and negative values of TSE can be observed without damping $(\xi=1)$. The oscillation is minimal for a damping

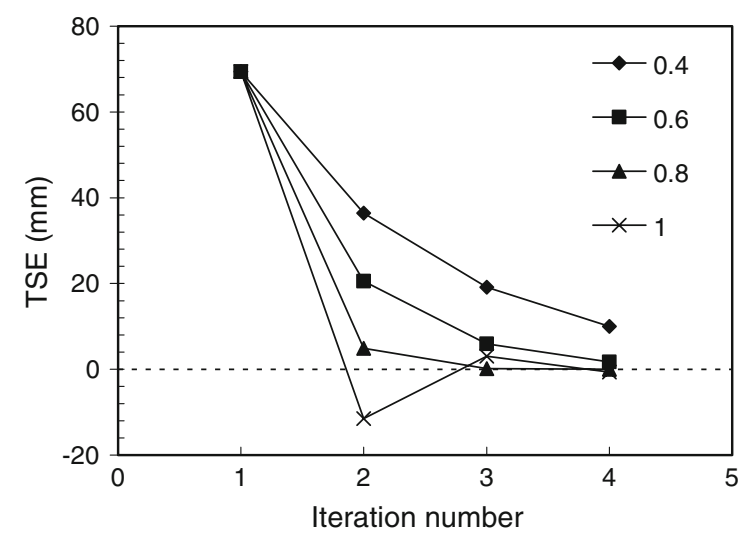

Fig. 8 Influence of damping coefficient on TSE evaluated with DD3IMP procedure coefficient of 0.8 , in this part, which leads to the fast convergence of the TSE. Though a damping coefficient of 0.8 resulted in fast convergence for this shallow part, for deeper parts, the required TSE is achieved after more iterations. Generally, a damping coefficient of 0.6 leads to fast convergence of TSE in the blank shape optimization algorithm (Oliveira et al. 2009). The results on the influence of the damping coefficient presented in Fig. 8 were obtained with the optimization procedure implemented in DD3IMP. Similar results are obtained by the optimization procedure implemented in $\mathrm{ABA}$ QUS also.

Figure 9 presents the global geometrical shape error evolution for the DD3IMP procedure with different damping coefficients. The evolution for GSE is presented in order to highlight the difference between the GSE and TSE value. In fact a small TSE value can be associated with a non-optimal blank shape, which has an area inside the target equal to the area outside the target. Figure 9 also presents the GSE evolution for the optimization procedure implemented in ABAQUS, only for the damping coefficient value of 0.6 . From the figure it is possible to confirm that when the push/pull technique is applied both procedures converge in five iterations. The small differences in the GSE error predicted by both procedures can be associated with the number of control points employed.

The empirical procedure used to obtain the initial blank (Fig. 7) is based on area conservancy and assumes that the thickness in the part remains the same after deep drawing. In actual practice, the blank

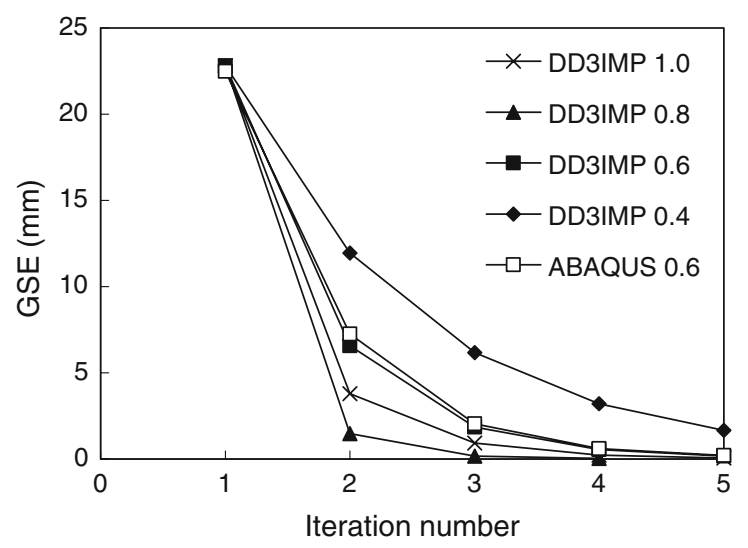

Fig. 9 Evolution of GSE using both optimization procedures 
is subjected to deformation process, and consequently to thickness variation. The result is an excess of material along the flange after deep drawing (see iteration 1 in Figs. 10 and 11). The proposed algorithm is then used to evaluate the differences between the flange contour and the target contour in subsequent iterations and accordingly modify the Bspline curve or NURBS surface, depending on the adopted procedure. The intermediate blank shapes used in deep drawing simulations resulted in the flange contour presented in Figs. 10 and 11 for the optimization procedure implemented in ABAQUS

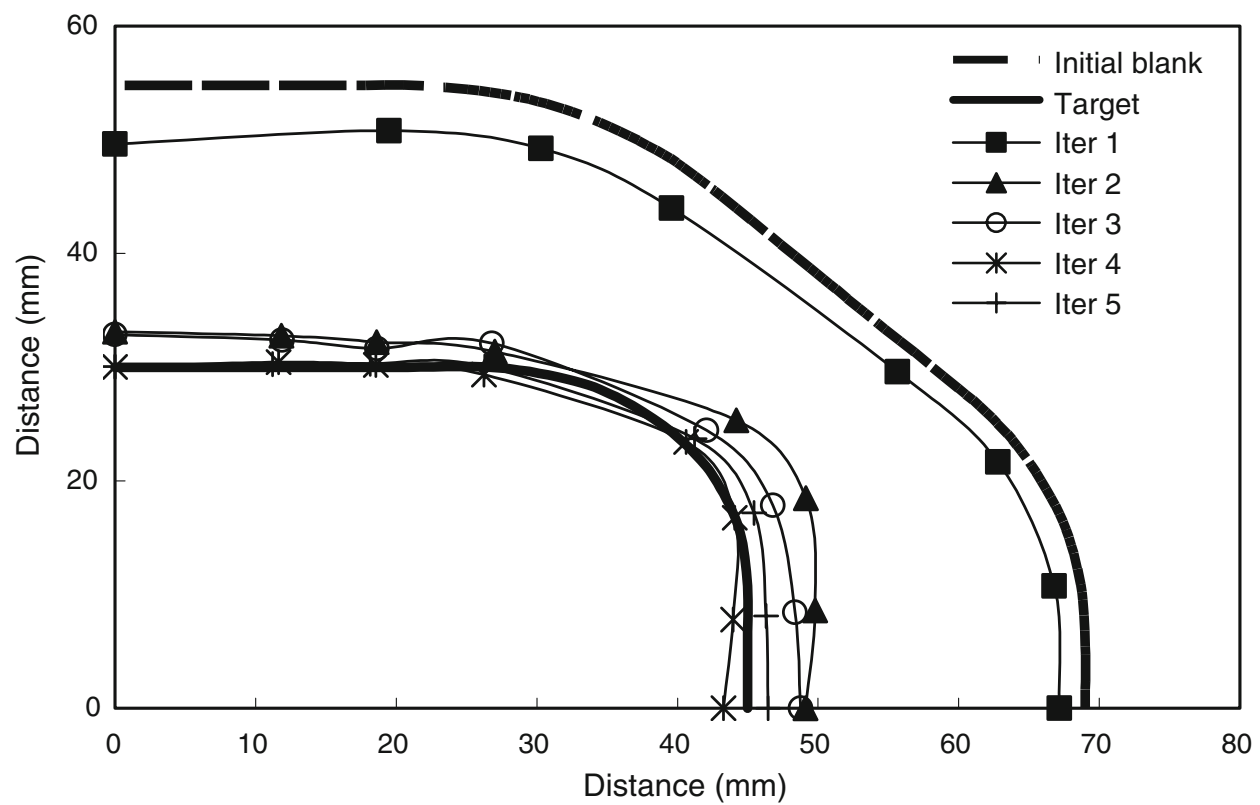

Fig. 10 Evolution of flange contour over iterations for the ABAQUS optimization procedure, using a damping coefficient of 0.6

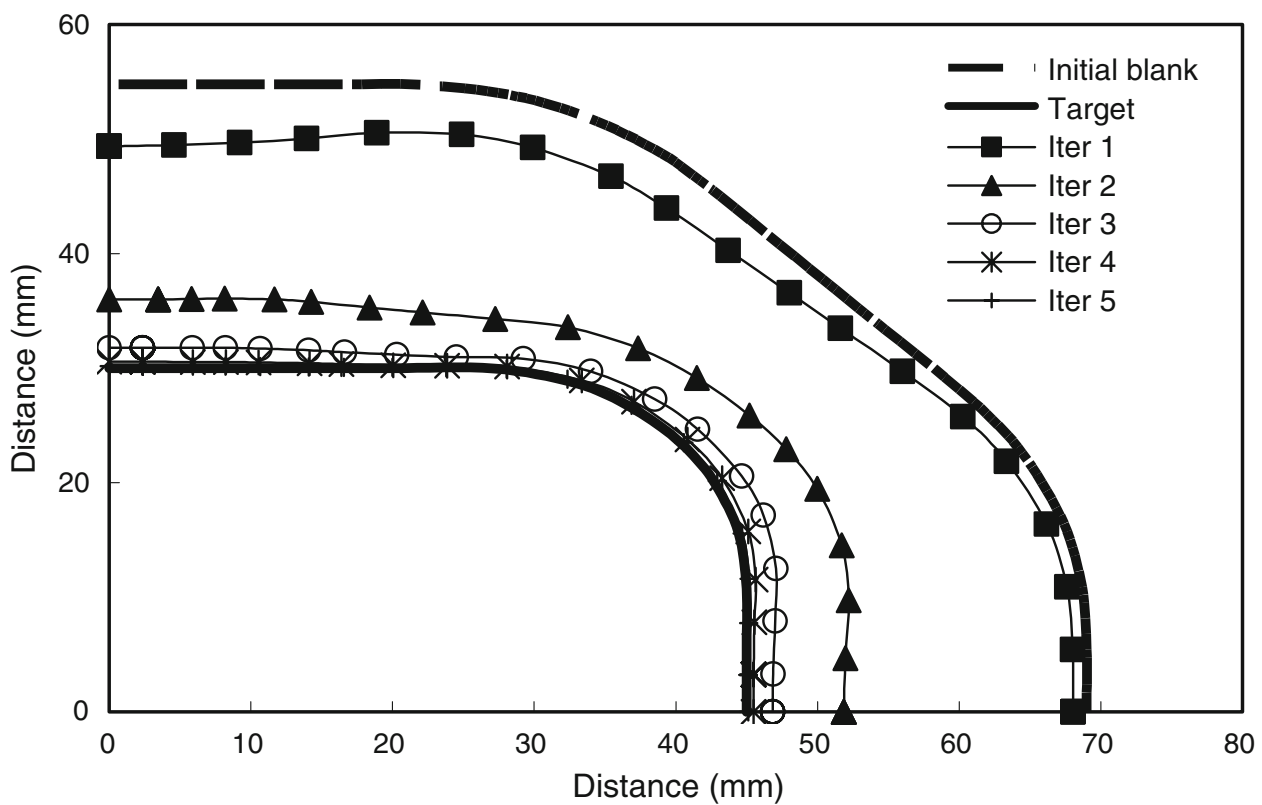

Fig. 11 Evolution of flange contour over iterations for the DD3IMP optimization procedure, using a damping coefficient of 0.6 
and DD3IMP, respectively. The initial blank is relatively large compared to the optimal blank and the drawing depth is only $14 \mathrm{~mm}$. Hence, the final contour after the first iteration is close to the initial blank contour as the draw-in is small.

As the number of iterations increase, the flange contour gets closer to the target contour. The shape error reaches a value of $0.204 \mathrm{~mm}$ within five iterations for the ABAQUS case, as shown in the Fig. 9, while it reaches 0.164 within five iterations for the DD3IMP case, as is also shown in Fig. 9. Any further iteration has negligible impact on the required flange contour as it depends on the finite element mesh density. However, a marginal difference between ABAQUS and DD3IMP flange contours can be observed. Comparatively, more material flow occurs in ABAQUS simulated cup than DD3IMP cup. Therefore, the flange contour in ABAQUS simulated cup is smaller than that obtained from DD3IMP simulation.

Figure 12 shows the thickness variation profile along OX direction in the rectangular cup at the end of first and last iteration of the blank shape optimization procedure, performed with ABAQUS and DD3IMP procedures. In the first iteration the blank size was large resulting in a large flange and hence the draw-in is comparatively less. Due to less drawin, maximum thinning occurred, especially at the punch radius. In the second iteration, a much smaller blank was utilised and consequently the thickness variation smoothed out due to a better draw-in. Thereafter, the thickness variation remained similar indicating that the chosen process parameters are optimal for the deep drawing process and results in uniform draw-in and marginal thickness variation in the rectangular cup. Marginal thickness increase was observed at the flange contour due to shear stress state.

Figure 13 shows the difference in the evolution of blank shape in both optimization procedures. Both procedures start with the same blank shape determined empirically. Marginal difference in the flange contour was observed at the end of the first iteration which persisted until the end of the optimization procedure, as shown in the figure. The differences in the blank shape can be attributed to the different FE mesh discretization used by ABAQUS and DD3IMP. ABAQUS uses a non-structured tetrahedral FE mesh while DD3IMP uses a structured hexahedral FE mesh with different mesh density that generates the small difference in the optimal blank shape. This leads to different thickness evolution in the blank, as illustrated in Fig. 11. As a consequence to better material flow prediction, better thickness distribution is obtained in the cup simulated using ABAQUS compared to DD3IMP. The difference can also be

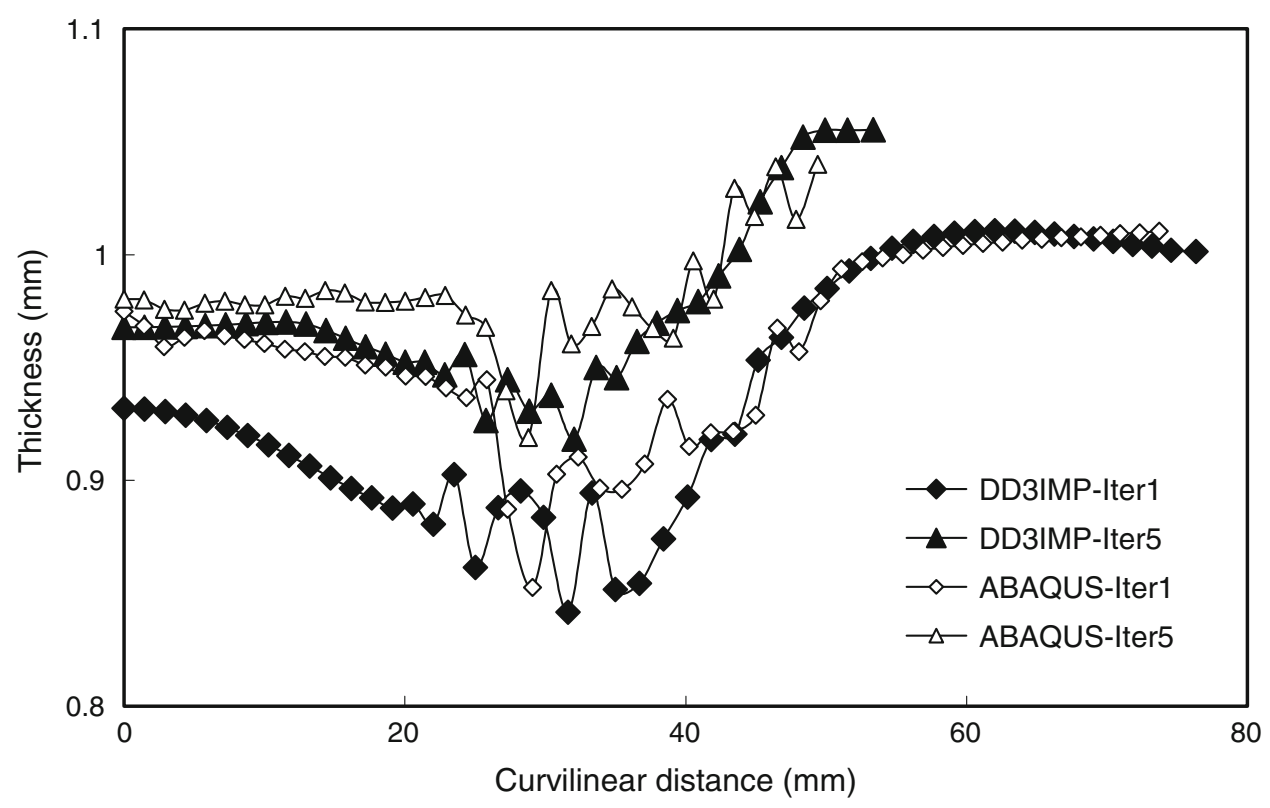

Fig. 12 Thickness evolution along OX during blank shape optimization procedure 


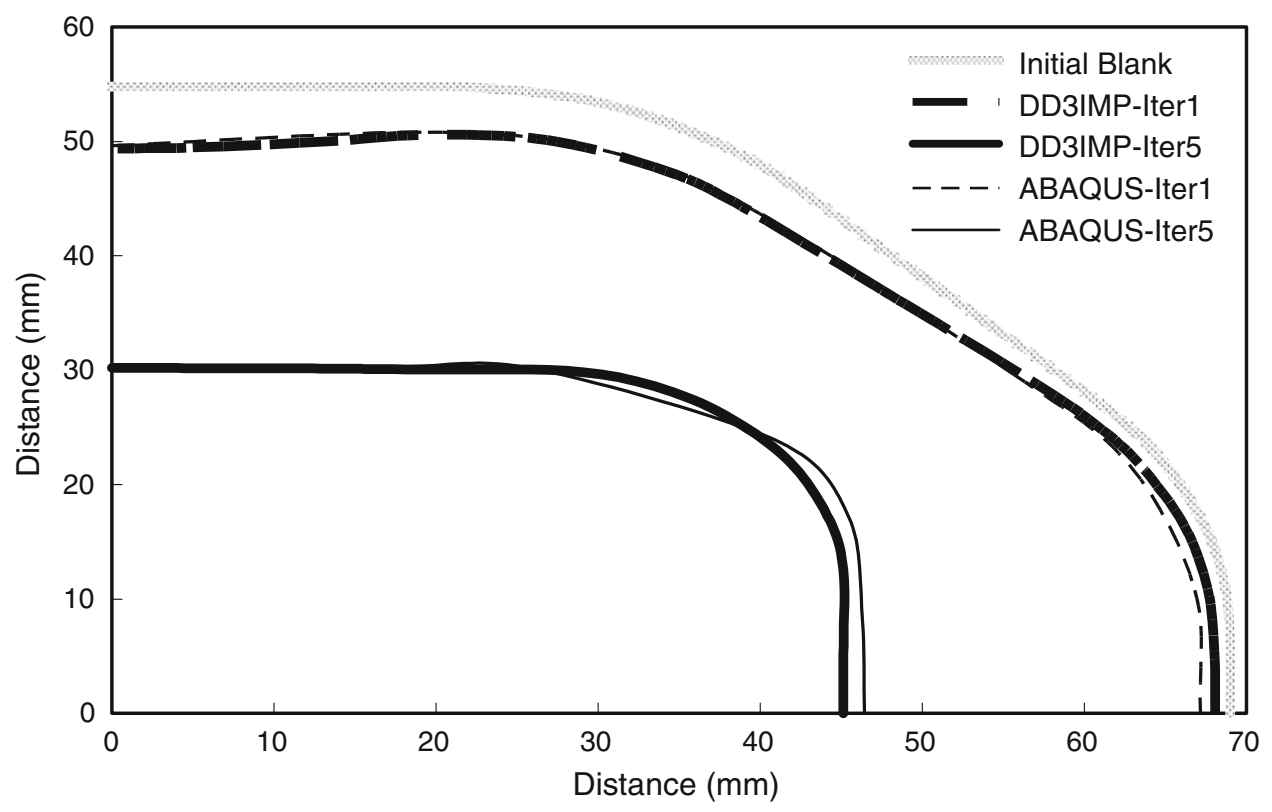

Fig. 13 Deformation behaviour

attributed to the number of control points used. DD3IMP procedure used 23 control points and hence has a smooth blank profile compared to ABAQUS procedure which used eight control points, hence the small difference in the optimal blank predicted by both methods.

An important aspect concerning optimization procedures is the computational effort. Due to the high difference in the number of degrees of freedom used in both codes, as shown in Fig. 7, no direct comparison on the computational effort is performed. Also, in the simulations performed with ABAQUS the automatic time stepping scheme is used. The number of increments in the simulations performed with ABAQUS is always lower than 130. In DD3IMP inhouse code, in each increment, an explicit approach is used to calculate an approximate first solution for the nodal displacements, the stress states and frictional contact forces. Then, a $r_{\text {min }}$ strategy is implemented to impose several restrictions on the size of the time increment in order to improve the convergence (Yamada et al. 1968). This first trial solution is iteratively corrected, using a Newton-Raphson algorithm, finishing when a satisfactory equilibrium state in the deformable body is achieved. It is then possible to update the blank sheet configuration, as well as all the state variables, passing on to the calculation of the next time increment. This is repeated until the end of the process (Menezes and Teodosiu 2000). The use of the $r_{\text {min }}$ strategy renders a variable number of increments, according with the material flow conditions. Nevertheless, the number of increments in the simulations performed with DD3IMP is always lower than 190. The small number of iterations required by the push/pull technique allows the optimization procedure of the rectangular cup to be performed with both FE codes in less than $12 \mathrm{~h}$, including the pre and post-processing procedures.

\section{Conclusions}

Blank design is an important task in sheet metal forming because it reduces the scrap, defects and improves the part quality. A deformation based blank shape optimization procedure involving push/pull technique is presented in this paper. The push/pull technique is applied to two different FEA codes, namely DD3IMP and ABAQUS, and the procedures are compared for their performance. The push/pull technique is an effective approach to determine the optimal blank shape for a part. Both the procedures predicted the optimal blank shape for a rectangular cup within few iterations. Marginal difference in the 
blank shapes predicted by the procedures can be attributed to the number of control points to which the push/pull technique is applied and to the mesh sensitivity. Hence, the presented blank shape optimization method, using deformation history and the push/pull technique, can be effectively used with any FEA software for quick prediction of an optimal blank for a part.

Acknowledgments The authors are grateful to the Portuguese Foundation for Science and Technology (FCT) for the financial support for this work, through the Program POCI 2010.

\section{References}

Baptista, A.J., Alves, J.L., Rodrigues, D.M., Menezes, L.F.: Trimming of 3D solid finite element meshes using parametric surfaces: application to sheet metal forming. Finite Elem. Anal. Des. 42, 1053-1060 (2006)

Barata da Rocha, A., Ferreira Duarte, J.: Tecnologia da Embutidura. Associacao Portuguesa das Tecnologias de Conformacao Plastica (APTCP) (ed.) (1993)

Guo, Y.Q., Batoz, J.L., Naceur, H., Bouabdallah, S., Mercier, F., Barlat, F.: Recent developments on the analysis and optimum design of sheet metal forming parts using a simplified inverse approach. Comput. Struct. 78, 133-148 (2000)

Hill, R.: A theory of the yielding and plastic flow of anisotropic metals. Proc. R. Soc. Lond. A 193, 281-297 (1948)
Kim, J.-Y., Kim, N., Huh, M.-S.: Optimum blank design of an automobile sub-frame. J. Mat. Process Tech. 101, 31-43 (2000)

Kuwabara, T., Si, W.-H.: PC-based blank design system for deep drawing irregularly shaped prismatic shells with arbitrarily shaped flange. J. Mat. Process Tech. 63, 89-94 (1997)

Li, K.P., Carden, W.P., Wagoner, R.H.: Simulation of springback. Int. J. Mech. Sci. 44, 103-122 (2002)

Menezes, L.F., Teodosiu, C.: Three-dimensional numerical simulation of the deep-drawing process using solid finite elements. J. Mat. Process Tech. 97(1-3), 100-106 (2000)

Oliveira, M.C., Alves, J.L., Menezes, L.F.: Algorithms and strategies for treatment of large deformation frictional contact in the numerical simulation of deep drawing process. Arch. Comput. Method E. 15(2), 113-162 (2008)

Oliveira, M.C., Padmanabhan, R., Baptista, A.J., Alves, J.L., Menezes, L.F.: Sensitivity study on some parameters in blank design. Mater. Design. 30(4), 1223-1230 (2009)

Padmanabhan, R., Oliveira, M.C., Baptista, A.J., Alves, J.L., Menezes, L.F.: Blank design for deep drawn parts using parametric NURBS surfaces. J. Mater. Process. Tech. 209, 2402-2411 (2009)

Park, S.H., Yoon, J.W., Yang, D.Y., Kim, Y.H.: Optimum blank design in sheet metal forming by the deformation path iteration method. Int. J. Mech. Sci. 41, 1217-1232 (1999)

Piegl, L.A., Tiller, W.: The NURBS Book, 2nd edn. SpringerVerlag, Berlin (1997)

Yamada, Y., Yoshimur, N., Sakurai, T.: Plastic stress-strain matrix and its application for the solution of elastic-plastic problems by the finite element method. Int. J. Mech. Sci. 10(5), 348-354 (1968) 